\title{
A 5-year analysis of rapid response system activation at an in-hospital haemodialysis unit
}

\author{
S Galhotra, ${ }^{1}$ M A DeVita, ${ }^{2,3}$ M A Dew, ${ }^{2}$ R L Simmons ${ }^{3}$
}

${ }^{1}$ Albert Einstein College of Medicine and Montefiore Medical Center, Bronx, New York, USA

${ }^{2}$ University of Pittsburgh, Pittsburgh, Pennsylvania, USA

${ }^{3}$ University of Pittsburgh Medical Center Presbyterian Hospital, Pittsburgh, Pennsylvania, USA

\section{Correspondence to} Dr Michael A DeVita, 125 Dewey Street, Pittsburgh, PA 15218-1407 USA; mdevpgh@yahoo.com

Accepted 15 November 2009 Published Online First 16 June 2010

\begin{abstract}
Objective To study the incidence, patient and event characteristics, and outcome of rapid response system (RRS) activation on an in-hospital haemodialysis unit.

Design Retrospective review of all RRS events on an in-hospital 10-bed haemodialysis unit over a 64-month period (November 2001 to February 2007).

Setting University of Pittsburgh Medical Center Presbyterian Hospital, a 730-bed academic, urban, tertiary care adult hospital in the USA.

Interventions None.

Results Over a 64-month-period, 107 of 8928 patients undergoing haemodialysis on the dialysis unit required an RRS activation (12 events/1000 patients dialysed). The most common reasons for RRS activation were respiratory distress/hypoxaemia (27\%) and mental status change (24\%). Predictors of in-hospital mortality included old age (33\% in-hospital mortality for patients aged 65 years or older vs $14 \%$ for patients aged less than 65 years; $\left.\chi^{2}=5.66, d f=1, p=0.017\right)$, and RRS activation due to a respiratory abnormality $37 \%$ mortality for respiratory codes vs $18 \%$ for all other codes; $\chi^{2}=4.12, \mathrm{df}=1$, $\mathrm{p}=0.042$ ). Surprisingly, only $71 \%$ of the patients who had an RRS event had the event as dialysis was occuring. Twenty-four patients (22\%) met one or more RRS activation criteria upon first vital sign check in the dialysis unit; RRS was activated on $12(11 \%)$ of these patients before dialysis was started. Nineteen (18\%) additional patients had an RRS event after their dialysis session had ended, while awaiting transport back to their unit. Conclusions From our findings, it can be suggested that critical events often occur before and after dialysis treatment, during or awaiting transport. Careful assessment of these high-risk patients before and after transport, to and from the dialysis unit may be warranted.
\end{abstract}

Compared with the general inpatient population, patients with renal failure requiring haemodialysis have a higher morbidity and mortality. ${ }^{1}$ Dialysis patients consume more critical care resources, whether in the form of intensive care unit (ICU) stay or in the form of rapid response system (RRS) activation for urgent unmet critical care needs. Some other aspects of the inpatient care of dialysis patients are unique. For example, unlike other floor patients, in-hospital haemodialysis patients must travel to and from specialised haemodialysis units, thereby distancing them from active care givers and augmenting potential problems with handoffs.

The analysis of RRS activation in the general inpatient population has been successfully applied to improve patient safety and outcomes, contributing to the widespread adoption of this safety initiative. $^{2-14}$ However, RRS activation in dialysis patients has not been studied before, and we hypothesised that such an analysis might reveal unique systemic problems.

A preliminary review of RRS activation at our 10-bed in-hospital haemodialysis unit was conducted as part of routine review of all RRS activations in the hospital. We expected haemodynamic and electrolyte changes occurring during the process of dialysis itself to account for a large majority of RRS activations; however, the pilot data revealed that respiratory abnormality and systems issues were fairly common. We therefore performed a retrospective review of RRS activations at our 10-bed in-hospital haemodialysis unit over a prolonged (64-month) period to study the incidence, causes, safety issue characteristics and outcome of all RRS activations. The quality improvement and patient safety problems encountered are identified, and possible approaches to overcome them are discussed.

\section{METHODS \\ Hospital setting}

The University of Pittsburgh Medical Center Presbyterian Hospital is an adult, 730-bed (including 160 ICU beds with automated electronic cardiac rhythm/haemodynamic monitoring and a dedicated 1:2 nurse-to-patient ratio, 330 beds with electronic cardiac rhythm/pulse monitoring and 1:4 nurse-topatient ratio, and 240 beds with no automated electronic rhythm/pulse monitoring and a 1:6 nurse-to-patient ratio) university-based tertiary care hospital located in Pittsburgh, Pennsylvania, USA. At our institution, there is a well-established RRS that is composed of four components: (1) an afferent component, which consists of event detection and response triggering; (2) an efferent component, which involves crisis response in the form of a medical emergency team; (3) a patient safety and process improvement component; and (4) an administrative component that implements and sustains the other three components. ${ }^{15}$ Dialysis personnel, like anyone else in the hospital, have immediate access to a medical emergency team by activating the RRS. As previously described, our medical emergency team brings a team of physician and non-physician critical care professionals to the bedside to provide expert care in a crisis situation and to appropriately triage the patients thereafter. ${ }^{16} 17$ The medical emergency team is led by a critical care medicine faculty member and is available $24 \mathrm{~h}$ a day, 7 days a week. The responding team is the same as cardiac arrest team in composition and resources, but criteria for call type (cardiac arrest or condition ' $\mathrm{A}$ ' call, and other crises or condition ' $\mathrm{C}$ ' call) vary, and have been reported elsewhere. ${ }^{17}$ 
Haemodialysis inpatients comprise two groups of patients: (1) those dialysed at the bedside (ICU patients and some floor patients in whom transportation to the dialysis unit is logistically difficult or is inadvisable given the critical nature of their illness) and (2) those transported to a specialised inpatient dialysis unit that has nursing and physician staff who are available 24 h a day, 7 days a week. Our analysis was focused on patients being dialysed on our 10-bed specialised dialysis unit and excluded patients treated in the ICU or on the floor.

\section{Data collection}

The hospital 'code' database was used to identify all RRS activations over a 64-month period (November 2001 to February 2007). The code database is a log of all cardiopulmonary arrest and patient crisis calls. Each time the RRS is activated, information about the patient, RRS call type (arrest or crisis event), date, time and location is recorded by a hospital operator. This information is entered into a database for record-keeping and quality improvement purposes.

Our Hospital Patient Safety Committee routinely reviews all cardiopulmonary arrest events, and a large convenience sample of RRS crisis calls. For the purpose of this study, electronic patient medical records of all 107 patients who coded on the dialysis unit were reviewed to extract information about patient/event characteristics and outcomes. Each event was presented as a separate case to the code review committee. Discussions were focused on patient safety issues and event/ hospitalisation outcomes. Our aim was to better understand the processes of care so that appropriate improvements could be made to improve outcomes. All relevant patient data were entered into an Excel database (Microsoft Corporation, 2008; Microsoft, Redmond, Washington, USA).

\section{Statistical analysis}

Data were analysed using SPSS V.14 statistical software (SPSS Corporation, 2006; SPSS, Chicago, Illinois, USA). All reported $p$ values are two-tailed and those $<0.05$ were considered significant.

This project was approved by the Total Quality Council, which typically grants approval for Quality Improvement projects at our institution.

\section{RESULTS}

Eight thousand nine hundred and twenty-eight patients were dialysed 28886 times in the dialysis unit during the 64-month period (average of 3.2 dialysis sessions per patient). During the same period, 107 patients had 118 RRS events (12 events per 1000 patients dialysed and 4.1 events per 1000 haemodialysis sessions). Four of these patients suffered a cardiopulmonary arrest (0.14 arrests per 1000 haemodialysis sessions), three of whom died within $24 \mathrm{~h}$ and one survived to hospital discharge. For a detailed review of patient and event characteristics, only the index event $(\mathrm{N}=107)$ was considered for analysis. In-hospital mortality for the dialysis subgroup (22\%) was nearly six times that of the general inpatient population (3.5\%) at our hospital over the period. Relevant demographic and outcome characteristics for these patients are listed in table 1.

Table 2 lists the primary reasons for RRS activation among the 107 patients. The most common reason for RRS activation was respiratory distress and/or hypoxaemia (27\%).

Other relevant characteristics of these patients are shown in table 3. Twenty-four patients arrived to the haemodialysis unit meeting one or more criteria for RRS activation upon the first vital sign check. In 12 of these patients, RRS was triggered before haemodialysis was begun. Another 19 patients had their
Table 1 Demographic and outcome characteristics of patients with rapid response system activation on the haemodialysis unit $(N=107)$

\begin{tabular}{|c|c|}
\hline Variable & $\begin{array}{l}\text { Mean (SD) } \\
\text { (range) }\end{array}$ \\
\hline Age (years) & $62(16)(19-93)$ \\
\hline Weight (kg) & $76(23)(35-165)$ \\
\hline \multirow[t]{2}{*}{ Hospital length of stay (days) } & $25(29)(0-205)$ \\
\hline & $\mathrm{n}(\%)$ \\
\hline \multicolumn{2}{|l|}{ Gender } \\
\hline Male & $49(46)$ \\
\hline Female & $58(54)$ \\
\hline \multicolumn{2}{|l|}{ Ethnicity } \\
\hline European American & $66(62)$ \\
\hline African American & $39(36)$ \\
\hline Hispanic American & $1(1)$ \\
\hline Asian American & $1(1)$ \\
\hline \multicolumn{2}{|l|}{ Co-morbidities } \\
\hline Hypertension & $77(72)$ \\
\hline Diabetes & $54(51)$ \\
\hline Coronary artery disease & $46(43)$ \\
\hline Transplant & $32(30)$ \\
\hline Kidney & 16 \\
\hline Liver & 8 \\
\hline Heart & 5 \\
\hline Lung & 2 \\
\hline Multivisceral & 1 \\
\hline \multicolumn{2}{|l|}{ Outcome of hospital admission } \\
\hline Died during hospital stay & $24(22)$ \\
\hline Discharged to home & $35(33)$ \\
\hline Discharged to nursing home or SNF & $30(28)$ \\
\hline $\begin{array}{l}\text { Discharged to long-term acute care } \\
\text { facility }\end{array}$ & $13(12)$ \\
\hline Transfer to another healthcare facility & $5(5)$ \\
\hline
\end{tabular}

SNF, skilled nursing facility.

RRS activation after the dialysis session had ended and while they were awaiting transportation back to their inpatient unit (table 3).

Analysis of demographic and event variables with the outcome variables revealed that among the 107 patients with RRS activation, older patients were more likely to die during the hospitalisation (33\% in-hospital mortality for patients aged $\geq 65$ years vs $14 \%$ for patients aged below 65 years; $\chi^{2}=5.66$, $\mathrm{df}=1, \mathrm{p}=0.017$ ), although both groups had a high likelihood of

Table 2 Primary reason for RRS activation on the haemodialysis unit $(\mathrm{N}=107)$

\begin{tabular}{lc}
\hline Variable & $\mathbf{n}(\%)$ \\
\hline Primary reason for RRS activation & \\
Respiratory & $29(27)$ \\
$\quad$ Respiratory distress and/or hypoxaemia & \\
Neurological & $26(24)$ \\
Mental status change & $5(5)$ \\
Seizure & \\
Cardiovascular & $12(11)$ \\
Hypotension & $10(9)$ \\
Hypertension & $9(8)$ \\
Atrial fibrillation or flutter & $7(7)$ \\
Chest pain & $1(1)$ \\
Symptomatic bradycardia & \\
Other & $4(4)$ \\
Cardiopulmonary arrest & $3(3)$ \\
Uncontrolled bleeding & $1(1)$ \\
Dialysis catheter dislodgment &
\end{tabular}


Table 3 Other relevant characteristics of patients with RRS activation on the haemodialysis unit $(\mathrm{N}=107)$

\begin{tabular}{|c|c|}
\hline Variable & n (\%) \\
\hline \multicolumn{2}{|l|}{ Primary service at time of code event } \\
\hline General internal medicine & $49(46)$ \\
\hline Transplant surgery & $12(11)$ \\
\hline Renal medicine & $8(7)$ \\
\hline Cardiology & $8(7)$ \\
\hline Vascular surgery & $5(5)$ \\
\hline $\begin{array}{l}\text { Other (general surgery, plastic surgery, physical medicine and } \\
\text { rehabilitation, psychiatry, cardiac surgery, emergency medicine, } \\
\text { neurosurgery, pulmonary medicine, family medicine, thoracic } \\
\text { surgery, urology, geriatrics or critical care medicine) }\end{array}$ & $25(24)$ \\
\hline \multicolumn{2}{|l|}{$\begin{array}{l}\text { Was the haemodialysis session stopped or } \\
\text { postponed after RRS activation? }\end{array}$} \\
\hline Yes & $78(73)$ \\
\hline No & $5(5)$ \\
\hline Not applicable (dialysis never started or already completed) & $24(22)$ \\
\hline \multicolumn{2}{|l|}{ Triage after event } \\
\hline Transfer to intensive care unit & $75(70)$ \\
\hline Transfer to monitored bed & $17(16)$ \\
\hline Transfer back to original inpatient unit & $11(10)$ \\
\hline Transfer to operating room & $2(2)$ \\
\hline Death during event & $2(2)$ \\
\hline \multicolumn{2}{|l|}{ Patient status upon arrival to haemodialysis unit } \\
\hline $\begin{array}{l}\text { Unstable (meeting one or more code criteria during first vital } \\
\text { signs check on haemodialysis unit) }\end{array}$ & $24(22)$ \\
\hline Stable & $83(78)$ \\
\hline \multicolumn{2}{|l|}{ When was the code called? } \\
\hline Before haemodialysis begun & $12(11)$ \\
\hline During haemodialysis & $76(71)$ \\
\hline After haemodialysis completed & $19(18)$ \\
\hline
\end{tabular}

death as compared with the general hospital population (3.5\%). Those who had an RRS activation because of a respiratory abnormality (hypoxaemia and/or respiratory distress) were also more likely to die during hospitalisation compared with haemodialysis patients with neurological, cardiovascular or other symptoms triggering an RRS activation (37\% mortality for respiratory events vs $18 \%$ for all other events; $\chi^{2}=4.12, \mathrm{df}=1, \mathrm{p}=0.042$ ).

\section{DISCUSSION}

To our knowledge, this is the first study to date to report on RRS events and outcomes in an in-hospital dialysis unit. We found that dialysis patients had a high RRS activation rate and a nearly six times higher mortality rate than the general in-hospital patient population during the same period. The mortality rate is similar to that reported in the dialysis literature (220.5 per 1000 patient years at risk). ${ }^{1}$ Our cardiopulmonary arrest rate was 0.14 per 1000 haemodialysis sessions. This rate is double that reported in the dialysis literature, ${ }^{18}$ although our absolute incidence of arrests $(n=4)$ is too small to reach any definitive conclusions.

Respiratory distress and hypoxaemia were the most common reasons for RRS activation, and we found that they, along with older age, predicted higher short-term mortality. Older age is a known risk factor for mortality in dialysis patients. ${ }^{18}$ Higher mortality in patients with an RRS event due to a respiratory abnormality may be attributable to severe underlying intrinsic lung disease or infectious complications such as pneumonia. Researchers from other settings have also found significant respiratory abnormalities to be surrogate markers of poor outcomes, although these studies were not focused on dialysis patients. ${ }^{19-21}$ Wang et al reported that nearly $17 \%$ of patients with acute respiratory compromise developed a cardiopulmonary arrest. Survival to discharge was lower for cardiopulmonary arrest patients (14.3\%) than non-cardiopulmonary arrest patients $(58.4 \%)$, which we have also previously reported in our population. ${ }^{22}$ Quach et $a l^{20}$ have reported 38\% mortality in patients with RRS activation due to respiratory distress. Cretikos et $a l^{21}$ have found that abnormal respiratory rate might be the vital sign with the best combination of sensitivity and specificity when it comes to predicting a major adverse event in the near future of a floor patient. Future studies can shed light on the aetiopathophysiological basis of high mortality with respiratory complications in dialysis patients.

Analyses of processes of care and patient safety issues revealed suboptimal triage to and from the dialysis unit as an area worthy of a patient safety intervention. Twenty-four patients were found to meet one or more RRS activation criteria on the first vital sign check upon arrival at the dialysis unit. In half of these patients, the RRS was activated before dialysis was ever begun because they needed immediate critical care attention at the bedside. The other 12 patients were sick enough that they met RRS activation criteria but were treated using available resources and personnel only to require an RRS activation later. These 24 patients raise an important patient safety concern because they deteriorated before leaving their inpatient unit, in transport or on arrival to the haemodialysis unit. To improve safety and help understand the causes of 'predialysis deterioration', a vital sign check immediately before leaving for the dialysis unit might trigger a needed response sooner and help identify how deteriorations evolve. Similarly, the 19 patients who coded after their dialysis session had ended (some of whom had been waiting for more than $1 \mathrm{~h}$ ) could benefit from more prompt transportation and closer attention to their vital signs before they deteriorate enough to necessitate RRS activation.

Thus, our data suggest that dialysis patients may benefit from closer monitoring and care in the hours before, during and after their dialysis session. While automatic vital sign monitoring on the dialysis beds helps track physiological parameters and early detection of clinical deterioration, closer attention to physiological parameters and critical care needs in the hours before and after dialysis sessions (including during their intra-hospital road trip) may help prevent adverse outcomes in a significant percentage of haemodialysis patients that decompensate before or after their treatment on the unit.

Our study has several limitations. We report RRS dialysis events and outcomes at a single institution. It is possible that other tertiary care hospitals might have different patterns of results based on the difference in the characteristics of their patient population and/or processes of care. However, the demographic, morbidity and mortality data of our population are similar to that reported by the United States Renal Data System ${ }^{123}$; major differences, therefore, are unlikely. We focused our analysis on patients being dialysed on the specialised dialysis unit and excluded patients who received in-hospital dialysis in other settings like the ICU; our findings may not be applicable to this latter group. An additional limitation pertains to the possibility that although we captured all the RRS activations on the dialysis unit, there may have been instances where a critical care physician treated a patient but an RRS was not activated. Another limitation is that events may have had nothing to do with the scheduled dialysis treatment since some of these patients may have met RRS activation criteria while on their respective inpatient units or deteriorated en route to the haemodialysis unit. We included these patients in our study 
group because of the temporal and physical proximity to the dialysis unit; however, they may represent a group of patients whose event had nothing to do with dialysis. For example, the events may be a result of faulty transport processes. We recognise this limitation and feel that our data nevertheless show that dialysis patients are at risk for critical deteriorations and have high in-hospital mortality.

In conclusion, patients with an RRS event on the in-hospital haemodialysis unit had high mortality, and were more likely to die in the hospital if they were older (age $\geq 65$ years) or had respiratory symptoms triggering the RRS activation. Careful assessment of these high-risk patients before and after transport, to and from the dialysis unit may be warranted.

Competing interests None

Provenance and peer review Not commissioned; externally peer reviewed.

\section{REFERENCES}

1. United States Renal Data System. USRDS 2008 Annual Data Report. http://www. usrds.org/2008/ref/G Morbidity \& Hospitalization 08.pdf and http://www.usrds. org/2008/ref/H Mortality \& Causes of Death 08.pdf (accessed Jul 2009).

2. Braithwaite $\overline{\mathbf{R S}}$, DeVita $\overline{M A}$, Mahidhara R, et al. Medical Emergency Response Improvement Team (MERIT). Use of medical emergency team (MET) responses to detect medical errors. Qual Saf Health Care 2004;13:255-9.

3. Rapid response teams: the case for early intervention. Institute for Healthcare Improvement. http://www.ihi.org/lHI/Topics/CriticalCare/IntensiveCare/ ImprovementStories/RapidResponseTeamsTheCaseforEarlylntervention.htm (accessed Jul 2009).

4. Critical care outreach 2003: progress in developing services. UK: Department of Health. http://www.dh.gov.uk/en/Publicationsandstatistics/Publications/ PublicationsPolicyAndGuidance/DH 4091873 (accessed Jul 2009).

5. Buist MD, Moore GE, Bernard SA, et al. Effects of a medical emergency team on reduction of incidence of and mortality from unexpected cardiac arrests in hospital: a preliminary study. BMJ 2002;324:387-90.

6. Ball C, Kirkby M, Williams S. Effect of the critical care outreach team on patient survival to discharge from hospital and readmission to critical care: a non-randomized population based study. BMJ 2003:327:1014-16.
7. Bellomo R, Goldsmith D, Uchino S, et al. A prospective before-and-after trial of a medical emergency team. MJA 2003;179:283-8.

8. Pittard AJ. Out of our reach? Assessing the impact of introducing a critical care outreach service. Anaesthesia 2003:58:882-5

9. Bellomo R, Goldsmith D, Uchino S, et al. Prospective controlled trial of effect of medical emergency team on postoperative morbidity and mortality rates. Crit Care Med 2004;32:916-21.

10. DeVita MA, Braithwaite RS, Mahidhara R, et al. Medical Emergency Response Improvement Team (MERIT) Use of medical emergency team responses to reduce hospital cardiopulmonary arrests. Qual Saf Health Care 2004;13:251-4.

11. Garcea G, Thomasset S, Mcclelland L, et al. Impact of a critical care outreach team on critical care readmissions and mortality. Acta Anaesthesiol Scand 2004;48:1096-100.

12. Priestley G, Watson W, Rashidian A, et al. Introducing Critical Care Outreach: a ward-randomized trial of phased introduction in a general hospital. Intensive Care Med 2004;30:1398-404.

13. Sebat $\mathbf{F}$, Johnson D, Musthafa AA, et al. A multidisciplinary community hospital program for early and rapid resuscitation of shock in non-trauma patients. Chest 2005; 127:1729-43

14. Buist $\mathbf{M}$, Harrison J, Abaloz E, et al. Six year audit of cardiac arrests and medical emergency team calls in an Australian outer metropolitan teaching hospital. BMJ 2007:335:1210-12.

15. DeVita MA, Bellomo R, Hillman $\mathrm{K}$, et al. Findings of the first consensus conference on medical emergency teams. Crit Care Med 2006;34:2463-78.

16. Galhotra S, DeVita MA, Simmons RL, et al. Impact of patient monitoring on the diurnal pattern of medical emergency team activation. Crit Care Med 2006:34:1700-6.

17. Foraida MI, DeVita MA, Braithwaite RS, et al. Improving the utilization of medical crisis teams (Condition C) at an urban tertiary care hospital. J Crit Care 2003:18:87-94.

18. Karnik JA, Young BS, Lew NL, et al. Cardiac arrest and sudden death in dialysis units. Kidney Int 2001;60:350-7.

19. Wang HE, Abella BS, Callaway CW, et al. Risk of cardiopulmonary arrest after acute respiratory compromise in hospitalized patients. Resuscitation 2008;79:234-40.

20. Ouach JL, Downey AW, Haase M, et al. Characteristics and outcomes of patients receiving a medical emergency team review for respiratory distress or hypotension. $J$ Crit Care 2008;23:325-31.

21. Cretikos $\mathbf{M}$, Chen J, Hillman $\mathrm{K}$, et al. The objective medical emergency team activation criteria: a case-control study. Resuscitation 2007;73:62-72.

22. Galhotra S, DeVita MA, Simmons RL. Mature rapid response system and potentially avoidable in-patient cardiopulmonary arrests. Qual Saf Health Care 2007;16:260-5.

23. United States Renal Data System. http://www.usrds.org/2008/ref/ C Patient Characteristics 08.pdf (accessed Nov 2008). 